\title{
Evaluaciones económicas en salud: Conceptos básicos y clasificación
}

\author{
VÍCTOR ZARATE ${ }^{1}$
}

\section{Economic evaluations in healthcare: Basic concepts and classification}

The growing burden of healthcare costs has created an urgent need to economically appraise health interventions in order to prioritise those which offer better value for money in the local context. The aim of this article is to provide some basic principles of economic evaluations which will improve physicians' understanding of the methodology involved in this type of analysis and will also help to show how their

${ }^{1}$ Centre for Health Economics, The University of York, York, Y010 5DD, United Kingdom, email:vz503@york.ac.uk, Fax: $+44(0) 1904321402$ clinical practice relates to the economics of healthcare financing. Some of the main concepts describe is this paper include the definition and classification of economic evaluations in healthcare (i.e. Cost-minimization, cost-effectiveness, cost-utility and cost-benefit) and a brief description of a cost-effectiveness plane.

(Rev Med Chile 2010; 138 (Supl 2): 93-97).

Key words: Economic evaluation, classification, cost effectiveness.

\section{RESUMEN}

El alza creciente de los costos en salud ha creado la urgente necesidad de evaluar económicamente las intervenciones de salud con el objetivo de priorizar aquellas que ofrecen un mejor valor o beneficio en relación a sus costos en un contexto local. El propósito de este artículo es entregar algunos principios básicos de evaluaciones económicas que mejorarán el conocimiento médico acerca de la metodología utilizada en este tipo de análisis y además ayudará a demostrar como la práctica clínica se relaciona con la economía del financiamiento en salud. Alguno de los conceptos que se describen en el manuscrito incluyen la definición y clasificación de las evaluaciones económicas en salud (ej. Costo-minimización, costo-efectividad, costo-utilidad $y$ costo-beneficio) así como una breve descripción del plano de costo-efectividad.

Palabras clave: evaluación económica, clasificación, plano costo-efectividad

\section{Introducción}

El uso de evaluaciones económicas para apoyar la toma de decisiones en salud es una práctica que ha tomado cada vez más fuerza a nivel mundial. Esto se debe a que en la gran mayoría de los sistemas de salud existe la necesidad de generar una provisión de servicios sanitarios de calidad que contenga una demanda potencialmente ilimitada en un contexto de recursos escasos ${ }^{1}$.

En este sentido el análisis económico represen- ta un valioso mecanismo que mejora la eficiencia de los procesos de distribución presupuestaria entre los distintos niveles de atención en salud.

A nivel mundial países como Australia, Canadá e Inglaterra han integrado por muchos años la metodología económica como pilar fundamental en la toma de decisiones en salud e inclusive han creado organismos gubernamentales que regulan y aconsejan la adopción de nuevas tecnologías o medicamentos basados en criterios de costoefectividad ${ }^{2-4}$. 
El presente artículo pretende revisar alguno de los aspectos centrales que componen una evaluación económica sanitaria con el fin de aclarar conceptos y servir de guía práctica a aquellos investigadores que estén interesados en desarrollar este tipo de estudios en el país.

\section{Definición de una evaluación económica}

Se define como evaluación económica a una técnica cuantitativa desarrollada por economistas que permite evaluar programas que generalmente son de financiamiento público ${ }^{5}$. Originalmente se aplicaron en áreas como transporte o medio ambiente y solamente durante los últimos 20 a 30 años han tenido un auge en salud.

El principal propósito de una evaluación económica es promover el uso más eficiente de los recursos en un ambiente de escasez. De esta manera, el sistema de salud es considerado como una serie de procesos en los cuales las materias primas (hospitales, doctores, enfermeras, equipos médicos, medicamentos, etc) son transformadas en productos sanitarios (prestaciones de salud, programas sanitarios, calidad de vida, etc) que son finalmente consumidos por los potenciales clientes (población). Promoviendo la existencia de un mayor número de procesos eficientes presentes en el sistema, en desmedro de los que no lo son, el beneficio neto en salud aumenta.

Las estimaciones de costos y efectividad clínica que poseen las distintas intervenciones en salud pueden provenir de variadas fuentes siendo las más comunes los ensayos clínicos controlados, los estudios quasi-experimentales y los estudios observacionales. Es importante aclarar que las evaluaciones económicas en salud se enfocan en las mediciones de efectividad y no eficacia, ya que lo relevante es la magnitud del efecto de una determinada intervención al ser implementada en condiciones rutinarias. Una alternativa o complemento valido a los estudios clínicos ya descritos es el uso de modelos analíticos. Los modelos analíticos permiten generar predicciones empíricamente comprobables sobre determinados outcomes clínicos basados en una estructura teórica que habitualmente se construye con datos que provienen de distintas fuentes. Los modelos analíticos más usados en evaluaciones económicas son los árboles de decisión y los modelos de Markov.
En términos prácticos, una evaluación económica se define como un análisis comparativo de cursos alternativos de acción en términos de sus costos y consecuencias ${ }^{6}$. Su resultado principal se expresa como la razón incremental de costo-efectividad, que se describe con el siguiente algoritmo:

$$
\Delta C_{i} / \Delta E_{i}=\frac{C_{1}-C_{2}}{E_{1}-E_{2}}
$$

Donde:

$C_{1}$ y $E_{1}$ representan los costos y efectos del programa de mayor efectividad (normalmente el programa nuevo) y

$C_{2}$ y $E_{2}$ representan los costos y efectos del comparador (normalmente el programa actual).

\section{Tipos de evaluaciones económicas}

Existen diversos tipos de evaluaciones económicas en salud, sin embargo, todas deben comparar al menos dos alternativas de intervención en términos de sus costos y efectividad. Los beneficios y costos a considerar en dicha comparación dependerán de la perspectiva de análisis que adopte el investigador. Las perspectivas que se reportan con mayor frecuencia en la literatura son las de la sociedad, del sistema de salud o del paciente. La elección de la perspectiva de análisis constituye un elemento crucial en el diseño de una evaluación económica y que en parte explica la existencia de discrepancias con respecto a la disposición de financiar distintas intervenciones preventivas $o$ curativas $^{7,8}$.

Es importante notar que si la evaluación económica no compara los costos y consecuencias de dos o más alternativas, esta debe denominarse como parcial. Las evaluaciones económicas parciales involucran los estudios de: 1) descripción de costos; 2) descripción de costo-consecuencia y 3 ) análisis de costos. La descripción de costos se caracteriza porque no compara cursos alternativos de acción siendo su principal propósito el reporte de costos asociados a una determinada intervención. La descripción de costo-consecuencia por otra parte, agrega a lo anterior la descripción de outcomes, sin embargo, tampoco considera la evaluación de alternativas terapéuticas. Finalmente, 
el análisis de costos, sí compara distintos cursos de acción, pero examinando solamente la relación entre costos en desmedro de las consecuencias ${ }^{6}$.

El término costo-efectividad se tiende a utilizar a veces de forma genérica para referirse a cualquier tipo de evaluación económica hecha en salud, sin embargo, desde un punto de vista técnico, existen cuatro tipos principales: análisis de costo-minimización, análisis costo-efectividad, análisis costo-utilidad y análisis costo-beneficio. Si bien todos estos análisis emplean una metodología similar en la estimación de costos, se diferencian en el método utilizado para estimar los beneficios ${ }^{9}$.

\section{Análisis costo-minimización}

El análisis de costo-minimización (ACM) compara exclusivamente los costos de dos intervenciones alternativas bajo el supuesto que ambas proveen un nivel de beneficio equivalente. Un ejemplo clásico es la comparación entre la hospitalización de pacientes con enfermedades crónicas estables en hospitales versus hospitalización domiciliaria. La evidencia del grado similitud en términos de efectividad puede provenir de datos primarios del mismo estudio o secundarios provenientes de un meta-análisis. En la práctica existen pocos ACM dada la dificultad de que dos intervenciones provean exactamente los mismos beneficios ${ }^{10}$.

\section{Análisis costo-efectividad}

En los análisis de costo-efectividad (ACE) los beneficios de las estrategias a evaluar no son equivalentes y son medidos en unidades naturales de morbilidad, mortalidad o calidad de vida. Dentro de las unidades más frecuentemente utilizadas están las muertes evitadas, los años de vida ganados, cambios en unidades de presión arterial o colesterol, cambios en escalas de dolor o cambios en escalas de calidad de vida relacionada con la salud $^{10,11}$.

Los ACE tienen la limitante de ser uni-dimensionales, es decir, evalúan sólo una dimensión de los beneficios. Esto no sólo dificulta el proceso de elección del outcome a evaluar, ya que se debe tratar de elegir al más representativo de la intervención, sino que además limita las posibilidades de comparación entre distintas intervenciones.

\section{Análisis costo-utilidad}

El análisis de costo-utilidad (ACU) es multidimensional ya que considera como beneficio una unidad común que considera tanto la calidad de vida como la cantidad o largo de vida obtenida como consecuencia de una intervención. Está característica permite comparar entre si, distintas intervenciones para distintos problemas de salud. Las unidades más conocidas y utilizadas para medir beneficios en los ACU son los años de vida ajustados por calidad (AVACs o QALYs), los años de vida ajustados por discapacidad (DALYs) y los años saludables equivalentes (HYE) ${ }^{6}$.

La construcción de medidas genéricas de outcomes como los QALYs, requiere que la valoración de beneficios este expresada en preferencias por estar en un estado de salud y no en otro. Este valor, que puede ser asignado a través de mediciones hechas a un individuo o a la sociedad en su conjunto, tiene su origen en la noción de "utilidad esperada" de la ciencia económica. Las utilidades pueden ser medidas de forma directa utilizando las técnicas de "standard-gamble" (SG), time trade off (TTO), o indirectamente utilizando una encuesta como la EQ-5D. En Chile las preferencias poblacionales en salud para el instrumento EQ-5D se encuentran disponibles desde el 2008 en el sitio web de la Superintendencia de Salud ${ }^{12}$.

\section{Análisis costo-beneficio}

Los análisis de costo-beneficio (ACB) requieren que las consecuencias de la intervención a evaluar sean expresadas en términos monetarios, lo que permite al analista hacer comparaciones directas entre distintas alternativas por medio de la ganancia monetaria neta o razón de costobeneficio. El hecho que tanto los beneficios como los costos estén expresados en una misma unidad facilita que los resultados finales sean analizados no sólo en el ámbito de la salud, sino que también en comparación a otros programas de impacto social como es el caso de la educación o el transporte publico ${ }^{6}$.

En términos generales existen tres métodos para asignar un valor monetario a beneficios en salud: capital humano, preferencias reveladas y disposición de pago o valoraciones de contingencia. Cuando se utiliza el método de capital humano, 
los beneficios se cuantifican en relación al cambio o mejora de la capacidad en la productividad de los individuos medida por ingresos económicos asociados a esa productividad. Los estudios de preferencias reveladas por otra parte, buscan inferir la valoración de la salud a partir de las decisiones que toman los individuos en la práctica. Por ejemplo, se puede determinar la equivalencia entre el valor asociado al riesgo de tener un accidente laboral y el nivel de ingresos en una determinada profesión. Finalmente, en las valoraciones de contingencia, los individuos deben responder cuanto están dispuestos a gastar para obtener un determinado beneficio en salud o evitar los costos de una determinada enfermedad ${ }^{6}$.

\section{Plano de costo-efectividad}

Los resultados de una evaluación económica pueden representarse gráficamente a través del plano de costo efectividad, el cual expresa en el eje ' $\mathrm{X}$ ' el efecto en salud y en el eje ' $\mathrm{Y}$ ' el costo asociado. Si se asume que la terapia tradicional se encuentra en el origen, al realizar una comparación con una nueva intervención se generan 4 posible situaciones: 1) Que la nueva terapia sea más efectiva y menos costosa; 2) Que la nueva terapia sea más efectiva pero más costosa; 3) Que la nueva terapia sea menos efectiva pero menos costosa y 4) Que la nueva terapia sea menos efectiva y más costosa. Si estos escenarios de costo-efectividad se correlacionan con los puntos cardinales se obtienen respectivamente los cuadrantes $\mathrm{NO}, \mathrm{NE}, \mathrm{SO}$ y SE (Figura 1).

Los cuadrantes NO y SE se denominan con frecuencia como dominantes, ya que existiría una tendencia clara a adoptar o rechazar la nueva intervención. En cambio en los cuadrantes NE y SO existiría un grado de incertidumbre en cuanto a si el efecto extra vale el costo adicional asociado, o si la reducción del efecto es aceptable dada la disminución de costo que se produce al adoptar el nuevo tratamiento ${ }^{6}$. El nivel de costos y efectos que son establecidos como aceptables para un determinado sistema de salud se denomina "umbral". El umbral se representa a través de una razón que tiene en su numerador los costos monetarios y en el denominador una medida de ganancia en salud ${ }^{13}$. En el ejemplo descrito en la Figura 1, todas las intervenciones cuya relación incremental de

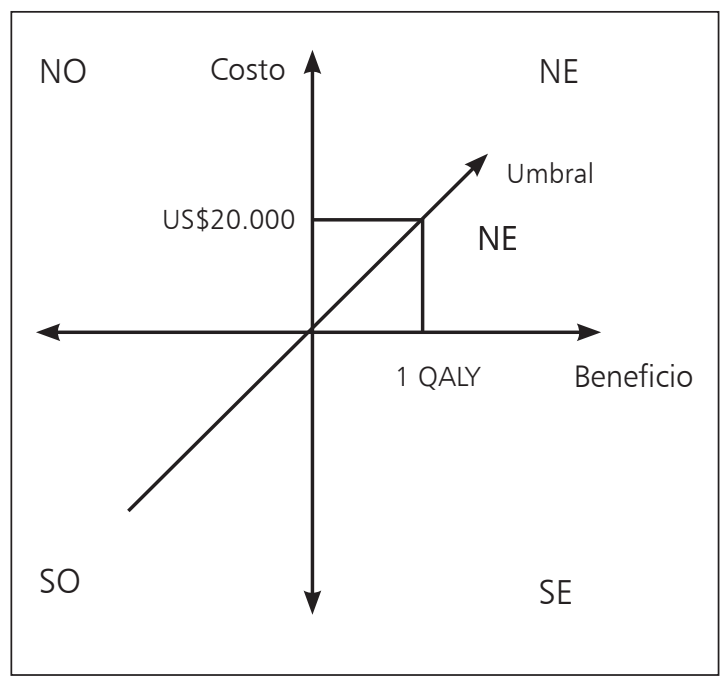

Figura 1. Plano de costo-efectividad.

costo-efectividad sea igual o menor a $\$ 20.000$ por QALY, se ubicarán en el umbral o la derecha de la línea del umbral, y por ende serán consideradas costo-efectivas.

\section{Referencias}

1. Drummond MF, Cooke J, Walley T. Economic evaluation in healthcare decision-making: evidence from the UK. York: University of York Centre for Health Economics; 1996.

2. Australian Pharmaceutical Benefits Advisory Committee. Disponible en: http://www.health.gov.au/internet/ wcms/publishing.nsf/Content/PBS+Committees-1. [Consultado el 23 de Noviembre de 2005].

3. National Association of Pharmacy Regulatory Authorities. Disponible en: http://www.napra.org/. [Consultado el 23 de Noviembre de 2005].

4. National Institute for Clinical Excellence. Disponible en: http://www.nice.org.uk. [Consultado el 23 de Noviembre de 2005].

5. Raftery J. Economic evaluation: an introduction. BMJ 1998; 316 (7136): 1013-4.

6. Drummond MF, O’Brien B, Stoddart GL, Torrance GW. Methods for the economic evaluation of health care programs. 20 edición Oxford: Oxford University Press; 1997.

7. Meltzer MI. Introduction to health economics for physicians. Lancet 2001; 358 (9286): 993-8. 
Evaluaciones económicas en salud: Conceptos básicos y clasificación - V. Zarate

8. Byford S, Raftery J. Perspectives in economic evaluation. BMJ 1998; 316 (7143): 1529-30.

9. Torgerson D, Raftery J. Economics notes: measuring outcomes in economic evaluations. BMJ 1999; 318 (7195): 1413.

10. Goodacre S, McCabe C. An introduction to economic evaluation. Emerg Med J 2002; 19 (3): 198-201.

11. Palmer S, Byford S, Raftery J. Economics notes: types of economic evaluation. BMJ 1999; 318 (7194): 1349.
12. Superintendencia de Salud. Valoración social de los estados de salud EQ-5D en la población de la RM de Chile 2008. Disponible online en: http://www.supersalud.cl/documentacion/569/articles-5214_recurso_1.pdf [Último acceso 6 Julio 2008).

13. Eichler HG, et al. "Use of Cost-Effectiveness Analysis in Health-Care Resource Allocation Decision-Making: How Are Cost-Effectiveness Thresholds Expected to Emerge?” Value in Health 7, no. 5 (2004): 518-28. 